\title{
ESTRATEGIAS QUE FACILITAN LA ORGANIZACIÓN DEL TRABAJO ESCOLAR CON LOS NIÑOS Y NIÑAS QUE PRESENTAN TRASTORNOS DE LA ATENCIÓN
}

STRATEGIES THAT FACILITATE THE ORGANIZATION OF THE SCHOOL WORK WITH THE STUDENTS WHO PRESENT DISORDERS OF THE ATTENTION

\author{
Volumen 11, Número 3 \\ Setiembre-Diciembre
}

pp. 1-18

Este número se publicó el 15 de diciembre de 2011

Carmen María Cubero Venegas

La revista está indexada en los directorios:

LATINDEX, REDALYC, IRESIE, CLASE, DIALNET, DOAJ, E-REVIST@S,

La revista está incluida en los sitios:

REDIE, RINACE, OEI, MAESTROTECA, PREAL, HUASCARAN, CLASCO 


\title{
ESTRATEGIAS QUE FACILITAN LA ORGANIZACIÓN DEL TRABAJO ESCOLAR CON LOS NIÑOS Y NIÑAS QUE PRESENTAN TRASTORNOS DE LA ATENCIÓN

\author{
STRATEGIES THAT FACILITATE THE ORGANIZATION OF THE SCHOOL WORK WITH \\ THE STUDENTS WHO PRESENT DISORDERS OF THE ATTENTION
}

\author{
Carmen María Cubero Venegas ${ }^{1}$
}

\begin{abstract}
Resumen. Este artículo está sustentado en los resultados de un estudio realizado en el Instituto de Investigación en Educación de la Universidad de Costa Rica ${ }^{2}$, cuyo propósito fue, a partir de un proceso de investigaciónacción, conocer y reconocer aquellas estrategias que utilizan las maestras y los maestros para favorecer el aprendizaje significativo en estudiantes con trastornos de la atención. El proceso investigativo permitió sistematizar lo observado en el aula y a partir de esa realidad proponer algunos referentes teóricos que expliquen y den una base científica al personal docente para construir mejores herramientas para realizar su práctica docente y facilitar de esta manera el aprendizaje en las personas con trastornos de la atención, que por sus características requiere una estructura que guíe y apoye su desarrollo personal y cognitivo. La sistematización de las estrategias está basada en tres niveles en que las maestras y maestros pueden organizar su intervención, a saber, un nivel preventivo, un nivel de apoyo y un nivel de reorientación conductual o correctivo.
\end{abstract}

Palabras claves: TRASTORNOS DE ATENCIÓN, DÉFICIT DE ATENCIÓN, ENSEÑANZA Y APRENDIZAJE, NIVELES DE INTERVENCIÓN.

\begin{abstract}
This article is sustained in the results of an research realized in the Institute of Investigation in Education of the University of Costa Rica which intention was, from a process of investigation-action, to know and to admit those strategies that the teachers use to favor the significant learning in students with disorders of the attention. The research process allowed to systematize the observed in the classroom and from this reality to propose some theoretical modals that explain and give a scientific base to the educational personnel in order that it manages to construct better tools to realize your educational practice and to facilitate hereby the learning in this population group, which for your characteristics needs a structure that guides and I support your personal and cognitive development. The realized systematizing is based on three levels in which the teachers can organize your intervention, namely, a preventive level, a level of support and a level or corrective of behavioral reorientation.
\end{abstract}

Key words: DISORDERS OF ATTENTION, DEFICIT OF ATTENTION, EDUCATION AND LEARNING, LEVELS OF INTERVENTION.

\begin{abstract}
1 Estudios de Maestría en Administración Pública en el Instituto Centroamericano de Administración Pública. Licenciada en Psicología, Profesora de Enseñanza Media ambos títulos de Universidad de Costa Rica. Profesora de la Escuela de Orientación y Educación Especial, de la Universidad de Costa Rica. Coordinadora del Programa para el Mejoramiento de los Procesos de la Enseñanza y Aprendizaje del Instituto de Investigación en Educación (INIE) de la Universidad de Costa Rica. Subdirectora del INIE.
\end{abstract}

Dirección electrónica: carmen.cubero@ucr.ac.cr

Artículo recibido: 18 de enero, 2010

Aprobado: 28 de noviembre, 2011

\footnotetext{
${ }^{2}$ Cubero y Nieto. (2006) Cómo facilitar el proceso de enseñanza y aprendizaje en estudiantes con trastornos de atención. Informe final. Instituto de Investigación en Educación. Universidad de Costa Rica.
} 


\section{Palabras introductorias}

Es importante conocer y reconocer las diferencias individuales de las y los estudiantes y, en el caso de aquellos con trastornos de la atención, es imprescindible la comprensión de lo que le sucede a quien lo padece. Esto lo reafirma muy bien Revilla (1998) cuando plantea que los estudiantes necesitan responder a un medio social cambiante y que exige, no solo la adquisición de conocimientos, sino también que esos conocimientos puedan ser utilizados en forma eficiente.

Las diferencias individuales son el eje central del proceso de desarrollo integral del estudiantado en su relación con el aprendizaje. Las áreas física, cognitiva y psicosocial o socioafectiva adquieren un papel especial en la práctica educativa y están constituidas por factores que no pueden ser ignorados cuando se trata de plantear estrategias que faciliten dicha práctica.

En el presente artículo se revisarán las condiciones que afectan cada una de estas áreas. En el área física se consideran aquellas condiciones relacionadas con la salud y la nutrición de los alumnos y las alumnas, puesto que su calidad - o la falta de ésta - repercute en la capacidad y forma de aprender, al igual que otras condiciones particulares del ambiente como la iluminación, la temperatura, el sonido, los ruidos, el consumo de alimentos, etc. Las determinaciones de tipo biológico, así como las reacciones a los diferentes elementos y estímulos varían de una persona a otra, pero es importante que el maestro y la maestra las tome en consideración cuando está tratando de comprender la vivencia del individuo con el que se relaciona cotidianamente.

En el área cognitiva, serán de especial atención para los maestros y maestras facilitadores de procesos de enseñanza y aprendizaje, las características particulares de los niños y las niñas en sus preferencias de tipo cognitivo para percibir, asimilar, procesar y transformar la información que recibe del medio. En el caso de los alumnos y alumnas con déficit de atención, el poder observar con detenimiento en cuál o cuáles partes de los procesos cognitivos presenta mayores dificultades, se convierte en un asunto de obligado compromiso.

No se puede dejar de mencionar, al pretender crear o utilizar estrategias que faciliten el aprendizaje, lo que Revilla (1998) menciona respecto a las características propiamente dichas de los estilos de aprendizaje, que el maestro y a maestra debe considerar si pretende coadyuvar a que los niños y niñas se desarrollen integralmente. Entre ellas cita el hecho de 
que el estilo de aprender de una persona puede ser diferente en situaciones diferentes. Aspectos como la edad, las circunstancias en que se enseña, el nivel de exigencia en la tarea por realizar y el tipo de aprendizaje a que se enfrenta la persona, inciden directamente en el rendimiento académico. Tanto Revilla (1998) como Butler, citado por Salas (1995), indican algunas ventajas que tiene el personal docente cuando logra adecuar el currículum escolar al estilo de aprendizaje que va desarrollando el niño y la niña y el grupo en general. Entre ellas señalan que el maestro y la maestra puede ayudar a orientar mejor el aprendizaje, utilizar mejor las estrategias para lograr el aprendizaje efectivo, favorecer el rendimiento académico y a partir de esto mejorar la autoestima y el autoconcepto, favorece la salud mental del alumnado porque aprende con menos tensión, y, agrega la autora de este artículo, conservar la salud mental de las personas docentes que puede ver a sus estudiantes alcanzar el éxito en la tarea propuesta, lo que sin lugar a dudas le provocará un sentimiento de bienestar y satisfacción profesional.

Por su parte, en el área psicosocial, aspectos como la motivación, la autoestima y el autoconcepto, tanto personal como académico del estudiante, inciden en el proceso de enseñanza y de aprendizaje. Para autores como Rossel (1998) una adecuada o inadecuada autoestima repercute de forma constante y persistente en el aprendizaje y el rendimiento escolar. La motivación hacia el estudio, según lo expuesto por Woolfolk (1999), incluye aspectos intrínsecos tales como la voluntad y el deseo de aprender, así como aspectos extrínsecos relacionados con la familia y el medio escolar y social, entre otros.

Desde la perspectiva social, como señala Dunn (1996), hay estudiantes a los que se les facilita el proceso de aprendizaje cuando pueden trabajar solos, mientras que otros funcionan mejor cuando pueden trabajar en grupo. Para una gran mayoría de los alumnos y alumnas con dificultades de atención, el trabajar con sus coetáneos les beneficia, siempre y cuando el docente pueda escoger adecuadamente a los participantes en el grupo, que deben ser alumnos capaces de manejar la estructura que requiere el trabajo en equipo, el seguimiento de instrucciones y la orientación a la tarea. El alumnado con estas características son una buena guía para mantener a los niños y niñas con trastornos de la atención centrados en el trabajo. Sin embargo, estudiantes con las características propias del déficit de atención, son poco demandados por sus iguales debido a que esas particularidades personales hacen más dificultoso el trabajo en grupo, suelen ser rechazados y no escogidos como compañeros de actividades escolares o recreativas. 
Como puede apreciarse, facilitar procesos de aprendizaje, va más allá de aspectos biológicos. Es más bien la combinación de elementos o características psicológicas, emocionales y fisiológicas, como señala Wilson (2002), “... cada uno de nosotros es afectado de manera diferente por características psicológicas, ambientales, físicas y sociales que intervienen en el proceso de concentración, aprendizaje y retención de información...". El juego entre factores como motivación, persistencia y responsabilidad se desarrolla según si las interacciones sociales son positivas o negativas. Lo anterior lleva a la reflexión de que plantear estrategias requiere conocimiento de la información en estudio, conocimiento de las particularidades, capacidades, habilidades y, destrezas del estudiantado, autoconocimiento del mismo docente respecto a su papel, a su concepción de estudiante, de aprendizaje y de evaluación, y a la satisfacción o insatisfacción que pueda tener de su desarrollo profesional. También es importante considerar las características propias del centro educativo y el entorno en que este se desenvuelve.

Resumiendo, es factible indicar que las situaciones de aprendizaje pueden ser consideradas y analizadas partiendo de ciertas premisas y que estas permiten el desarrollo de estrategias para su potencialización. Wilson (2002) señala algunas de ellas:

- Cada una de las personas tiene diferentes fortalezas

- Una gran mayoría de los individuos tiene capacidad para aprender

- Cada persona tiene capacidad de responder al ambiente de acuerdo con sus fortalezas

- Cada persona tiene preferencias individuales de instrucción y a partir de esto, se pueden establecer las mediciones de rendimiento.

- La mayoría de los docentes pueden aprender a usar los estilos de aprendizaje como un medio para facilitar el proceso de aprendizaje.

- Algunos estudiantes pueden aprender a capitalizar sus fortalezas si se les ayuda a reconocer el estilo de aprendizaje.

El y la docente pueden considerar aspectos relacionados con el manejo del ambiente y con lo sociológico, lo fisiológico y lo psicológico para lograr una exitosa atención de todos sus estudiantes y por supuesto, entre ellos aquellos que requieren una atención especial. 


\section{Consideraciones básicas al planear la cotidianeidad del aula}

En este apartado se va a privilegiar el aporte realizado por estudiosos de la práctica educativa, entre ellos el de Dunn (1996), Salas (1995) y Griggs y Dunn (1996). Se describen a continuación algunas de las consideraciones básicas que se deben tener en cuenta al planear una clase y al tratar de facilitar los procesos de atención. Estas consideraciones son generales, pero brindan, a juicio de la autora, un marco de referencia para el trabajo que los y las docentes desarrollan en la atención de los niños y niñas con trastornos de atención y de las cuales también se beneficia el resto del alumnado que comparte el proceso de aprendizaje. Es conveniente que el y la docente las considere cuando está planeando la lección, escogiendo la metodología, los materiales y el lugar de la clase en que el niño y la niña estarán ubicados.

2.1 Estímulos ambientales: se señalan aspectos tales como la intensidad de la luz, la temperatura, el sonido y el diseño de la sala de clase. Cada persona tiene su propia respuesta o gusto por el modo en que estos estímulos se presentan. A continuación una breve mención de ellos.

- Silencio vrs sonido: algunas personas pueden aprender aun cuando el nivel de ruido sea alto, mientras que para otras el sonido bloquea las actividades cognitivas que se requieren para concentrarse, prestar atención o memorizar y procesar la información. Mientras que algunos estudiantes requieren silencio para acciones como realizar la función ejecutiva del habla interna, para trasladar información de la memoria de trabajo a la memoria de largo plazo, otras por el contrario pueden lograrlo en ambientes ruidosos, sin problema. Generalmente las aulas son ruidosas, por lo que aquellos estudiantes que requieren mayor silencio, manifiesten o no trastornos de la atención, van a tener dificultades mayores para funcionar.

- Luz brillante vrs luz baja: la presencia o ausencia de iluminación es otro de los factores que afectan el rendimiento de algunos estudiantes. Mientras que unos funcionan mejor en ambientes muy iluminados, pues de lo contrario se sienten somnolientos, a otros la luz brillante les inquieta. En los niños y niñas con trastornos de la atención, al igual que a sus coetáneos, la intensidad de la luz es un factor que requiere observación, para poder ubicarlos físicamente en el aula, de acuerdo con su preferencia. 
- Frío vrs calor: las personas responden de manera diferenciada a la temperatura; por ejemplo, algunos estudiantes pueden responder mejor cuando el tiempo es cálido, otros por el contrario buscan sentarse cerca de puertas y ventanas donde manifiestan sentirse confortables. Las preferencias de temperatura deben acomodarse de tal manera que las necesidades individuales faciliten la eficacia en el aprendizaje.

- Disposición del aula: en este sentido, el docente debe tener presente que las comodidades en cuanto a tamaño del mobiliario, espacio vital mínimo, estado físico del mobiliario, cantidad de estudiantes en un determinado espacio físico, cantidad de materiales que debe manipular el estudiante, ubicación y estado de la pizarra o de los recursos de apoyo que utiliza el docente, organización del aula, de los pupitres o bien la decoración de la misma, son condiciones que afectan la capacidad de respuesta de los y las estudiantes a las situaciones de aprendizaje.

2.2 Estímulos emocionales: la manera como un estudiante se desenvuelve en el entorno educativo se relaciona directamente con el rendimiento académico que este logra alcanzar, de ahí la importancia de que los maestros y las maestras consideren los estímulos emocionales como factores determinantes en el proceso de enseñanza y de aprendizaje. En las personas con trastornos de la atención, estos estímulos se convierten en un valioso aliado del docente para lograr mantener de manera sostenida la motivación, la responsabilidad y la persistencia que se requieren para que atienda, comprenda y asimile los contenidos en estudio, así como para que pueda mantener adecuadamente su autoestima y autoconcepto.

- Motivación: para Borda y Pinzón (1995, p. 22), así como para Woolfolk (1999), la motivación “... es el centro productor de energía, que impulsa la acción, trabajo, estudio y la realización plena del ser humano". En el estudio realizado, la motivación es uno de los factores a los que el maestro debe dedicar más tiempo. Impulsar el proceso motivador, sostenerlo y lograr finalizar el objetivo propuesto, es una de las tareas y retos más importantes a los que se enfrentan los docentes en su labor cotidiana con los estudiantes y personalmente.

- Persistencia: este factor se relaciona directamente con la voluntad. La voluntad: el querer hacer o lograr un objetivo se convierte en el impulso inicial que debe ser mantenido, a partir de una actividad constante, en muchas ocasiones más allá de los 
límites previstos. La persistencia, también denominada en algunas ocasiones como la disciplina para el logro de la tarea, es uno de los aspectos que mayor dificultad provoca en el manejo o atención de los niños y niñas con trastornos de la atención. Al ser estos inconsistentes en su rendimiento y tener dificultad para mantenerse en una tarea el tiempo que esta requiere para que sea completada, particularmente, cuando se está ante actividades rutinarias o repetitivas, que son características del trabajo escolar.

- Responsabilidad: este aspecto es de observar con sumo cuidado al estar trabajando con personas con trastornos de atención. La mayoría de las veces cuando nos referimos a la responsabilidad se parte de la premisa de que la persona responsable es aquella que es cumplida, además de que lo hace con gusto, puntualidad, diligencia y que puede apreciarse un avance constante en su desarrollo personal. En el caso de las personas con trastornos de la atención, es consistente el hecho de encontrar que estas características no necesariamente son la respuesta esperada, aunque sí se encuentra, al menos en los niños y niñas que inician la escuela, una actitud tendiente a cumplir, solamente que no cuentan con los recursos personales para hacerlo.

- Estructura vrs flexibilidad: la estructura es el establecimiento de las normas de trabajo; buscan completar una tarea o bien el logro de los objetivos y cumplir con esos fines de una determinada manera y en un lapso definido. La flexibilidad, según lo plantean Dunn y Dunn, citados por Salas (1995), se refiere a la variación o cambios metodológicos y de los tiempos, entre otros factores, para proporcionar al estudiantado otros medios de cumplir con lo demandado. También en este aspecto podrían señalarse las adecuaciones curriculares o de acceso que suelen realizar los maestros y maestras para facilitar el proceso de aprendizaje de sus estudiantes.

En el caso de las personas con trastornos de la atención, estos aspectos deben ser administrados de manera equitativa, por un lado requieren de una estructura clara y consistente, pero a la vez flexible de manera que pueda ajustarse a sus características, siempre manteniendo en mente que ellos manifiestan inconsistencias en cuanto al rendimiento y a la calidad del trabajo, pero requieren una exigencia sostenida para que demuestren lo que pueden lograr. 
2.3 Estímulos sociológicos: en esta categoría se agrupan las condiciones sociales que prefiere el alumno para estudiar. Son variables, a veces depende de la materia en estudio o del trabajo que deba enfrentar, o bien en ocasiones se encuentran estudiantes que prefieren una sola modalidad.

- Trabajo individual: algunos estudiantes aprenden mejor cuando estudian solos pues esto les permite organizar su tiempo, trabajo y materiales a su manera. No es necesariamente la mejor manera de hacerlo para las personas con trastornos de la atención debido a su inconsistencia con respecto a las tareas que debe realizar y al cumplimiento de los objetivos propuestos. Sin embargo, cuando la persona requiere trabajar sola porque siente que se distrae más si lo hace en compañía de otros, (probablemente cuando esté más avanzado respecto a los primeros años de la educación escolar), entonces se le debe facilitar el trabajo de manera muy estructurada para que logre sostener la atención y la concentración en la tarea.

- Trabajo en equipo: cuando el estudiante se siente bien en un grupo en donde las tareas están equitativamente distribuidas se podría pensar en que el aprendizaje es una consecuencia lógica que le proporciona a la persona no solo conocimiento sino también un sentimiento de bienestar. Los y las estudiantes con trastornos de la atención, no necesariamente logran cumplir con las demandas del grupo de manera consistente, por lo que en muchas ocasiones, el trabajo en equipo se convierte en un elemento que les lesiona su autoestima al no poder cumplir en forma eficiente y a tiempo con lo que estas situaciones de aprendizaje requieren.

- Trabajo con la orientación de una persona adulta: la presencia de una persona adulta, (docente, padre, madre, etc.), puede ser un recurso que permita a los y las estudiantes sentirse seguros y guiados en su trabajo. No obstante, cuando los deseos de autonomía e independencia aparecen como características normales del desarrollo, entonces se manifiestan las dificultades para que esta orientación sea aceptada en buenos términos por la alumna o el alumno, por lo que sería un factor a considerar cuando el niño o la niña son pequeños, pero que no siempre es exitosa cuando son preadolescentes o adolescentes. Sin embargo, una persona que ejerza una función de tutoría, con especialización en la materia, que trabaje brindándole el apoyo requerido, en un ambiente dispuesto para tal fin, puede convertirse en un buen recurso. Probablemente en el ambiente familiar o escolar el estudiante se sienta presionado y 
esta tensión provoque rechazo que se puede manifestar desde negación de la tarea por realizar, hasta situaciones de verdadera violencia.

- Trabajo en pareja: este es quizá uno de los estímulos sociológicos que mejor se adaptan a los alumnos y las alumnas con trastornos de la atención, siempre y cuando la pareja sea un o una estudiante con una buena estructura y orientación a la tarea, como se mencionó líneas arriba. El tener cerca una persona que le guíe, que sea un buen modelo, es un recurso que se utiliza con frecuencia para apoyar a estudiantes con trastornos de atención.

Es frecuente que los estudiantes varíen estas modalidades sociológicas dependiendo de la materia en estudio, de la forma en que se le plantee el apoyo y de las características personales de los interlocutores. Son estímulos valiosos que deben ser adecuadamente administrados por el docente.

2.4. Estímulos psicológicos: en este grupo de estímulos, los autores mencionados incorporan las preferencias cognitivas de los estudiantes. Anteriormente se había hecho referencia a ellas de manera general; en este apartado se pretende desarrollarlas brevemente.

- Preferencias cognitivas analíticas o globales: hay estudiantes que en general abordan el conocimiento de manera analítica, se distinguen porque asimilan la materia de manera detallada, prácticamente juntando un hecho tras otro y prestan atención a aspectos relevantes, y no relevantes, que para ellos adquieren significado. Los estudiantes con trastornos de la atención que presentan dificultades para discriminar los aspectos importantes de aquellos que son accesorios, están ante una grave dificultad cuando tienen un docente con un estilo de enseñanza analítico pues se les dificulta establecer esas diferencias, por el contrario, si ambos -docente y estudiantecoinciden en su estilo de aprendizaje analítico esta situación se convierte en una ventaja.

Por otro lado, aquellos estudiantes con estilos de aprendizaje globales que se acercan al conocimiento a partir del dato general y no prestan mayor atención a los detalles, podrían encontrar dificultades en materias que requieren la discriminación cognitiva entre el conocimiento global y la desagregación de hechos que lo han conformado. 
Esto último puede ser una dificultad importante para el estudiante en materias, por ejemplo, como la matemática, la física o los estudios sociales que requieren la comprensión global pero sustentada en pequeños detalles.

- Nivel de impulsividad vrs reflexividad: en las personas con características de impulsividad, como es el caso de los y las estudiantes con trastornos de déficit de atención con o sin hiperactividad, el nivel de impulsividad es una seria limitación, no solo para funcionar cognitivamente en el medio escolar, sino también para mantener un comportamiento acorde con lo que se requiere en el contexto de un aula.

Retomando algunas de las características que distinguen a las personas con déficit de atención y las descritas por los autores señalados al inicio de este apartado, se encuentra que la premura con que dan las respuestas y la ocurrencia que está presente en la mayoría de sus intervenciones, provoca dificultades que se reflejan tanto en su rendimiento académico como en su autoestima. La dificultad para reflexionar, sobre todo antes a dar una respuesta, es una de las particularidades de las personas que presentan este trastorno.

Es conveniente mencionar que cuando se trata de valorar las acciones que una persona profesional en educación debe considerar para facilitar el aprendizaje del alumnado, particularmente de aquel que requiere una atención diferenciada en apoyo a sus necesidades especiales, es necesario tener presente la diferencia entre el comportamiento, adecuado o no, de los y las estudiantes y la sensibilización que a partir del conocimiento les permite dar un apoyo más respetuoso y eficiente a esta población. La capacitación constante que permita estar actualizados, que su práctica docente sea un proceso de investigación, reflexión y análisis, de manera que pueda mejorar y estar en constante aprendizaje a partir de su experiencia, son aspectos relevantes al plantear condiciones y estrategias alternativas para el trabajo en el aula.

La intervención en el aula, permite a los maestros y las maestras, así como a los niños y niñas con déficit de atención, trabajar en un ambiente estructurado, que facilite el aprendizaje, pero que a la vez les permita sentirse seguros y con posibilidades de ser exitosos. Cubero (2003, p. 3) señala que los niveles de intervención "corresponden a acciones que tienen una interdependencia entre sí; corresponde a un proceso de organización de la dinámica del aula. Se plantea en tres niveles: el preventivo, el de apoyo y el correctivo. Este modelo, en su propuesta inicial, se 
deriva de los estudios de Charles (1989) y ha sido trabajado en diferentes momentos por Cubero y Nieto (2003). Siguiendo a Cubero (2003) se conceptualiza cada nivel de intervención:

a) Nivel preventivo: busca anticipar las acciones para evitar que una conducta se manifieste o bien busca las acciones para que la conducta se incorpore o se mantenga. En el caso de los niños con trastornos de la atención, estos manifiestan conductas que necesariamente deben ser controladas o eliminadas y requieren incorporar otras y mantenerlas tanto para que tengan éxito en el trabajo académico como en las relaciones interpersonales. El maestro o la maestra deben pensar anticipadamente la forma que consideren es la más favorecedora del proceso de enseñanza y de aprendizaje para los niños y niñas con trastornos de atención, así como para el resto del alumnado. Esta intervención se origina en la necesidad de que tanto el acto educativo como el que provoca la vivencia aúlica se base en un pensamiento anticipatorio que les permite estar preparado para potencializar al máximo el proceso de aprendizaje.

b) Nivel de apoyo: busca que las conductas que se quieren evitar o eliminar sean excluidas mediante acciones de recordatorio o de aplicación directa de consecuencias. Asimismo, las conductas que se desean incorporar o mantener sean reforzadas oportunamente. Como particularidad, el nivel de apoyo permite que la lección se desarrolle sin interrupción, pues las acciones deben ser recordatorios previamente acordados entre el maestro o la maestra y el niño o la niña, o entre el grupo, el docente y el niño. Aquí se incluye todo refuerzo de carácter afectivo: sonrisas, palmadas suaves en la espalda o sobre la cabeza, caricias, palabras cariñosas, reconocimientos breves, etc. Permite al alumno desarrollar estrategias de autocontrol y no lesiona la autoestima, al evitar el llamado de atención puntual y constante.

c) Nivel de reorientación conductual o correctivo: busca enmendar el comportamiento inadecuado y reorientarlo. Nótese que no es solo corregir, pues tanto en el caso de los niños con trastornos en la atención como con los que no lo tienen, se da el caso de que se corrige la conducta que para el adulto es perturbadora o inadecuada, sin embargo, si no se trabaja la reorientación, la explicación y/o el modelaje de lo que se requiere, en muchas ocasiones el alumno no sabe qué es lo que se espera de él en ese momento y circunstancia. En el caso de que sea una conducta de eliminación o de incorporación que se había previsto en el nivel preventivo y se había apoyado debidamente, en el segundo 
nivel de intervención, entonces lo que cabe en este nivel es la aplicación de la consecuencia prevista, sin dejar por ello de reorientar, informar o recordar cuál es la conducta que se está esperando. En el caso de que sea una conducta inaceptable, no prevista oportunamente, esta debe detenerse de inmediato, aplicar una consecuencia razonable y adecuada a la falta y dejar muy claro cuál es la conducta que se espera en otra oportunidad.

Las estrategias que se presentan a continuación fueron trabajadas con el personal docente de una escuela pública, de nivel básico, ubicada en una zona urbana. Se basan en la propuesta de intervención organizada en los tres niveles anteriormente mencionados. Se debe aclarar que cada maestro o maestra las puede y debe adecuar según las características particulares de los niños y niñas y sus propias características personales, las oportunidades que tenga de desarrollar su práctica docente y el conocimiento que muestre sobre el trastorno de déficit de atención. No son una receta en el aula, en educación, como en prácticamente todas las áreas de la vida, las recetas no existen, incluso en la cocina, lo que diferencia la buena cuchara, es poder experimentar y que cada quien le agregue los ingredientes de manera individualizada. Solo así se pueden presentar sabores y olores para disfrutar todos los días. En el aula es igual, se deben buscar las condiciones, adecuar los factores mencionados y procurar dar oportunidades de éxito al estudiantado. 


\section{CUADRO 1}

\section{Estrategias dirigidas al trabajo con estudiantes con Déficit Atencional ${ }^{3}$}

\begin{tabular}{|c|c|c|}
\hline \multicolumn{3}{|c|}{ Por nivel de intervención } \\
\hline $\begin{array}{l}\text { Nivel de intervención } \\
\text { Preventivo }\end{array}$ & $\begin{array}{l}\text { Nivel de intervención de } \\
\text { Apoyo }\end{array}$ & $\begin{array}{l}\text { Nivel de intervención de } \\
\text { Reorientación Conductual o } \\
\text { Correctivo }\end{array}$ \\
\hline $\begin{array}{l}\text { Acordar conjuntamente señales y } \\
\text { claves para recordarle al estudiante } \\
\text { los comportamientos que debe } \\
\text { mantener. }\end{array}$ & $\begin{array}{l}\text { Utilizar las señales y claves acordadas para que el estudiante } \\
\text { vuelva a su trabajo }\end{array}$ & $\begin{array}{l}\text { Evaluar y retomar, en caso necesario, el } \\
\text { sistema de señales y claves. }\end{array}$ \\
\hline $\begin{array}{l}\text { Reconocer la dificultad de autocontrol } \\
\text { que tiene el estudiante en el salón de } \\
\text { clases. }\end{array}$ & $\begin{array}{l}\text { Ubicar al alumno con dificultades, cerca de estudiantes que } \\
\text { puedan ser un buen modelo, y lejos de aquellos con menor } \\
\text { autocontrol. }\end{array}$ & $\begin{array}{l}\text { Cambiar periódicamente la ubicación } \\
\text { física de los estudiantes en el aula }\end{array}$ \\
\hline $\begin{array}{l}\text { Mantener el contacto visual con el } \\
\text { estudiante para facilitar la } \\
\text { comunicación no verbal. }\end{array}$ & $\begin{array}{l}\text { Sentar al estudiante en un sitio en el cual pueda mantener el } \\
\text { contacto visual, particularmente cuando da instrucciones. }\end{array}$ & $\begin{array}{l}\text { Reestructurar el sistema de señales y } \\
\text { claves para facilitar la comunicación no } \\
\text { verbal como estrategia de autocontrol. }\end{array}$ \\
\hline $\begin{array}{l}\text { Reconocer que el alumno con } \mathrm{DA}^{4} \\
\text { puede parecer desconsiderado a } \\
\text { pesar de no tener esa intención }\end{array}$ & $\begin{array}{l}\text { Valorar los sentimientos e ideas de los estudiantes. } \\
\text { Mantenga el respeto por las diferencias individuales. } \\
\text { No permitir comportamientos inadecuados. }\end{array}$ & $\begin{array}{l}\text { Reflexionar con el estudiante sobre } \\
\text { otras alternativas de comportamiento } \\
\text { para relacionarse con las personas. }\end{array}$ \\
\hline $\begin{array}{l}\text { Identificar a los estudiantes que } \\
\text { necesitan apoyo extra, asistencia y } \\
\text { monitoreo cuidadoso fuera del aula }\end{array}$ & $\begin{array}{l}\text { Mantener en mente que los estudiantes con déficit de } \\
\text { atención, tienen mayores dificultades en ambientes no } \\
\text { estructurados. }\end{array}$ & $\begin{array}{l}\text { Aumentar la supervisión fuera del aula y } \\
\text { proveer más opciones de actividades, } \\
\text { por ejemplo en el recreo, en el comedor, } \\
\text { en el bus, entre otras. }\end{array}$ \\
\hline $\begin{array}{l}\text { Planear el uso de materiales y } \\
\text { estrategias que faciliten al estudiante } \\
\text { focalizar y mantener la atención. }\end{array}$ & $\begin{array}{l}\text { Hacer preguntas interesantes, utilizar cuadros, gráficos, } \\
\text { historias. Lectura de poemas. } \\
\text { Utilizar técnicas efectivas para realizar preguntas que } \\
\text { permitan que el estudiante piense y reflexione sobre el } \\
\text { trabajo que se está realizando. } \\
\text { Hacer la lección lo más participativa, amena y variada } \\
\text { posible. }\end{array}$ & $\begin{array}{l}\text { Llevar registros, observar y analizar el } \\
\text { resultado de las estrategias utilizadas, } \\
\text { de tal manera que identifique aquellas } \\
\text { de mayor éxito para mantener el interés } \\
\text { y la atención de los y las estudiantes. }\end{array}$ \\
\hline
\end{tabular}

\footnotetext{
${ }^{3}$ Discutidas y adecuadas con la M.Sc. Marisol Nieto, especialista en Educación Especial.

${ }^{4}$ DA significa déficit de atención.
} 


\begin{tabular}{|c|c|c|}
\hline & $\begin{array}{l}\text { Hacer uso de estrategias informales: juegos, } \\
\text { dramatizaciones, etc., para lograr la atención y el interés. } \\
\text { Agregar un poco de misterio, por ejemplo, traiga un objeto a } \\
\text { la lección, manténgalo en una caja y genere el interés del } \\
\text { alumno por el mismo. } \\
\text { Llamar la atención del estudiante de manera auditiva: uso de } \\
\text { campanas, reloj de cocina, etc. } \\
\text { Variar su tomo de voz, fuerte, suave, susurro. } \\
\text { Usar señales visuales: prenda y apague las luces o levante la } \\
\text { mano. Utilizar un foco para llamar la atención de los } \\
\text { estudiantes. } \\
\text { Encuadrar el material visual que quiere que los alumnos } \\
\text { observen utilizando sus manos o con un marco de color } \\
\text { alrededor. } \\
\text { Usar el color para llamar la atención: marcadores de colores, } \\
\text { lápices, tizas de colores, crayolas, etc. } \\
\text { Tener presente que usted debe ser un modelo de } \\
\text { entusiasmo. }\end{array}$ & \\
\hline $\begin{array}{l}\text { Enseñar utilizando estrategias que } \\
\text { permitan al estudiante sostener la } \\
\text { atención y la concentración. }\end{array}$ & $\begin{array}{l}\text { Moverse por el aula para mantener la visibilidad de los } \\
\text { estudiantes. } \\
\text { Buscar una forma de trabajar el tema por medio de unidades } \\
\text { que permitan la integración de ideas y conceptos. } \\
\text { Dar las lecciones siguiendo un ritmo vivaz y enérgico. } \\
\text { Utilizar técnicas de evaluación de aplicación de } \\
\text { conocimientos: hacer preguntas abiertas que requieren de } \\
\text { razonamiento, estimular el pensamiento crítico y las } \\
\text { discusiones. } \\
\text { Disminuir la cantidad de tiempo de sus intervenciones. } \\
\text { Aumentar la cantidad de respuestas de sus estudiantes. } \\
\text { Promover la constante participación de los estudiantes. } \\
\text { Recurrir al trabajo de grupo, tanto para el logro de objetivos } \\
\text { como para facilitar la atención sostenida en el tema, por } \\
\text { ejemplo: permitir que respondan a una pregunta al } \\
\text { compañero del lado, dibujar en conjunto la respuesta o bien } \\
\text { la preparación cooperativa de una respuesta escrita }\end{array}$ & $\begin{array}{l}\text { Recurrir a la estructura de grupos de } \\
\text { trabajo cooperativo. Darles oportunidad } \\
\text { de trabajo conjunto a los estudiantes } \\
\text { con déficit de atención, particularmente } \\
\text { si se les dificulta trabajar en situaciones } \\
\text { de poca estructura. }\end{array}$ \\
\hline
\end{tabular}




\begin{tabular}{|c|c|c|}
\hline $\begin{array}{l}\text { Pensar en forma positiva en las } \\
\text { necesidades particulares de los } \\
\text { estudiantes con déficit de atención. }\end{array}$ & $\begin{array}{l}\text { Cuidar el ambiente para que el estudiante se sienta seguro y } \\
\text { confortable en el aula, sabiendo que será tratado con } \\
\text { dignidad y respeto y que no será avergonzado ni humillado. } \\
\text { Garantizar que el estudiante se sienta confiado en que sus } \\
\text { maestros y maestras se preocupan por sus necesidades } \\
\text { particulares. } \\
\text { Crear espacios para que el estudiante pueda expresar } \\
\text { sentimientos, preocupaciones, temores e ideas. } \\
\text { Asegurar que el aula tenga una estructura positiva, y } \\
\text { acogedora que permita que el estudiante sentirse parte de } \\
\text { ella. }\end{array}$ & $\begin{array}{l}\text { Reafirmar al estudiante que tiene } \\
\text { alternativas y algún poder de decisión. }\end{array}$ \\
\hline $\begin{array}{llrr}\text { Reflexionar y analizar sobre } & \text { las } \\
\text { destrezas } & \text { sociales } & \text { que } & \text { usted } \\
\text { considera deseables para } & \text { una } \\
\text { convivencia armónica en el aula. } & \end{array}$ & $\begin{array}{l}\text { Dramatizar, argumentar y modelar las destrezas sociales que } \\
\text { espera que sus alumnos desarrollen. }\end{array}$ & $\begin{array}{l}\text { Discutir con los estudiantes la necesidad } \\
\text { de estas habilidades sociales cuando se } \\
\text { presenten comportamientos } \\
\text { inadecuados. } \\
\text { Dramatizar nuevamente la destreza, } \\
\text { cerciorándose de que los estudiantes la } \\
\text { están comprendiendo la propuesta } \\
\end{array}$ \\
\hline $\begin{array}{l}\text { Construir estrategias pedagógicas } \\
\text { considerando las áreas fuertes de los } \\
\text { estudiantes. }\end{array}$ & $\begin{array}{l}\text { Estimular y reconocer el logro alcanzado en todas las fases } \\
\text { del proceso de aprendizaje. }\end{array}$ & $\begin{array}{l}\text { Evaluar las estrategias para } \\
\text { enriquecerlas con materiales, técnicas y } \\
\text { recursos que faciliten el proceso de } \\
\text { aprendizaje. }\end{array}$ \\
\hline Dar oportunidades de logro. & $\begin{array}{l}\text { Diseñar actividades que permitan que el estudiante las pueda } \\
\text { realizar exitosamente. }\end{array}$ & $\begin{array}{l}\text { Reconstruir con el estudiante los } \\
\text { procesos de una tarea, asignación, etc., } \\
\text { destacando los logros y desaciertos en } \\
\text { el mismo. }\end{array}$ \\
\hline $\begin{array}{l}\text { Organizar adecuadamente el tiempo } \\
\text { considerando las posibilidades reales } \\
\text { de cumplimiento de los estudiantes }\end{array}$ & $\begin{array}{l}\text { Monitorear y supervisar al estudiante en el uso adecuado del } \\
\text { tiempo mientras desarrolla su trabajo. } \\
\text { Utilizar apoyos tales como relojes, carteles, programaciones } \\
\text { escritas, tarjetas de colores, de manera que el estudiante } \\
\text { pueda permanecer en el trabajo demandado. } \\
\text { Enseñar el uso de estrategias de administración del tiempo y } \\
\text { el uso de por ejemplo agendas, recordatorios, } \\
\text { establecimiento de prioridades, división del trabajo, etc. }\end{array}$ & $\begin{array}{l}\text { Reorganizar el trabajo del estudiante de } \\
\text { tal manera que pueda cumplir con los } \\
\text { objetivos básicos. } \\
\text { Retomar con el estudiante el proceso de } \\
\text { organización del tiempo seguido, para } \\
\text { ayudarle a tomar conciencia acerca de } \\
\text { cómo lo utilizó. }\end{array}$ \\
\hline Facilitar estrategias para el estudio & $\begin{array}{l}\text { Administrar algunos instrumentos básicos para conocer el } \\
\text { estilo de aprendizaje de los estudiantes. }\end{array}$ & $\begin{array}{l}\text { Valorar el rendimiento que ha tenido el } \\
\text { estudiante al utilizar las técnicas de }\end{array}$ \\
\hline
\end{tabular}




\begin{tabular}{|c|c|c|}
\hline & $\begin{array}{l}\text { Enseñar al estudiante aquellas técnicas de estudio que se } \\
\text { acerquen más a su estilo de aprendizaje. }\end{array}$ & $\begin{array}{l}\text { estudio recomendadas. } \\
\text { Reorientar su uso en caso de que sea } \\
\text { necesario. } \\
\text { Informar y explicar a los padres y } \\
\text { madres la utilidad que tiene para el } \\
\text { estudiante el seguir las técnicas } \\
\text { recomendadas. }\end{array}$ \\
\hline $\begin{array}{l}\text { Permitir que el estudiante utilice el } \\
\text { tiempo necesario para procesar la } \\
\text { información, producir y realizar las } \\
\text { actividades }\end{array}$ & $\begin{array}{l}\text { Dividir aquellos trabajos grandes en secciones de manera } \\
\text { que el estudiante pueda ir cumpliéndolas paulatinamente. } \\
\text { Seguir de cerca el trabajo que el estudiante está realizando } \\
\text { para monitorear el procesamiento de la información que está } \\
\text { haciendo. }\end{array}$ & $\begin{array}{l}\text { Readecuar el trabajo de manera que el } \\
\text { estudiante pueda ir comprendiendo el } \\
\text { contenido que está trabajando. } \\
\text { Reorientar el proceso de enseñanza y } \\
\text { de aprendizaje para el logro exitoso de } \\
\text { la comprensión. }\end{array}$ \\
\hline $\begin{array}{l}\text { Detectar señales que indiquen que el } \\
\text { alumno está empezando a } \\
\text { desconcentrarse. }\end{array}$ & $\begin{array}{l}\text { Cambiar el ritmo de la lección, utilizar estrategias novedosas, } \\
\text { incluso dar descanso si se percibe cansancio en los } \\
\text { estudiantes. }\end{array}$ & $\begin{array}{l}\text { Evaluar el trabajo para considerar si hay } \\
\text { mayor dificultad que la prevista o bien si } \\
\text { el trabajo es muy básico y tedioso para } \\
\text { el estudiante. } \\
\text { Revisar las metodologías empleadas en } \\
\text { la lección para enriquecerlas o } \\
\text { modificarlas. } \\
\text { Redirigir la situación asignando otras } \\
\text { tareas tales como ordenar libros de la } \\
\text { biblioteca, llevar y traer documentos, } \\
\text { enviarlo a hacer un mandado, etc. }\end{array}$ \\
\hline $\begin{array}{l}\text { Detectar las situaciones que provocan } \\
\text { conductas inapropiadas en los } \\
\text { estudiantes, por ejemplo hambre, } \\
\text { sueño, clases de determinada } \\
\text { materia, materiales, metodologías, } \\
\text { hora del día, etc., e iniciar un plan de } \\
\text { intervención preventiva. }\end{array}$ & $\begin{array}{l}\text { Aplicar las estrategias e innovar con ellas las lecciones. } \\
\text { Organizar los horarios, los cronogramas de trabajo } \\
\text { considerando las necesidades específicas de los estudiantes. } \\
\text { Permitir espacios lúdicos dentro del aula, relacionados con la } \\
\text { materia en estudio. } \\
\text { Utilizar el trabajo cooperativo en momentos en que los niños } \\
\text { estén más cansados, de manera que puedan "disfrutar" de la } \\
\text { compañía y el apoyo de los demás. } \\
\text { Cambiar el ambiente del aula, a partir de organizar de } \\
\text { manera diferentes los pupitres. }\end{array}$ & $\begin{array}{l}\text { Mantener una evaluación constante del } \\
\text { proceso de enseñanza de manera que } \\
\text { pueda detectar y valorar las variables } \\
\text { que pueden ocasionar mayores } \\
\text { conductas perturbadoras en los } \\
\text { estudiantes. }\end{array}$ \\
\hline
\end{tabular}




\section{A modo de reflexión final}

El trabajo realizado en las aulas permitió identificar muchas de las estrategias que se utilizan a diario para provocar situaciones de éxito en el estudiantado y facilitar el proceso de aprendizaje. También dicho trabajo permitió valorar el mejor desempeño docente y la mayor satisfacción en el mismo, cuando se logra estructurar el ambiente de manera que todos y todas, incluido el maestro y la maestra, están desarrollándose en un plano de respeto y consideración a las diferencias individuales.

Las condiciones que facilitan el aprendizaje en los ámbitos físico, sociológico y psicológico, son ampliamente reconocidas por las personas profesionales en educación, pero pocas veces aplicadas en la cotidianidad del aula para procurar mejores oportunidades al estudiantado. La incorporación de estas estrategias produjo un mayor bienestar y una efectiva preocupación por parte de la maestra y el maestro por el desempeño de los y las estudiantes. La previsión, el apoyo y la visión de reorientar la conducta y no solo sancionarla o señalarla, permitieron un cambio de actitud en la práctica docente y un ambiente realmente inclusivo, en el cual los niños y niñas identificados con trastornos de la atención, recibieron el apoyo necesario, sin discriminación y con la posibilidad de crear mecanismos de autocontrol en el comportamiento y satisfacción, al poder cumplir con las demandas que el medio escolar les plantea.

\section{Referencias}

Charles, Carol M. (1989). Building Classroom Discipline. New York: Longman Publishers USA. Thirh edition.

Charles, Carol M. (1996). Building Classroom Discipline. New York: Longman Publishers USA. Fifth edition.

Cubero, Carmen. (2003). Niveles de intervención en el aula para la atención de las personas con trastornos de la atención. Costa Rica: Revista Electrónica Actualidades Investigativas en Educación, 2 (1). Disponible en http://revista.inie.ucr.ac.cr/buscararticulos/controlador/Article/accion/show/articulo/niveles-de-intervencion-en-el-aula-parala-atencion-de-estudiantes-con-trastornos-de-atencion.html.

Cubero, Carmen y Nieto, Marisol. (2005). Cómo facilitar el proceso de enseñanza y aprendizaje en las personas con trastornos de atención (Informe de Investigación). San José: Instituto de Investigación en Educación. Universidad de Costa Rica. Costa Rica. 
Cubero, Carmen, Abarca, Angelina y Nieto, Marisol. (1996). Percepción y Manejo de la Disciplina en el Aula. Costa Rica: Universidad de Costa Rica (Informe de Investigación). San José: Instituto de Investigación en Educación. Universidad de Costa Rica. Costa Rica.

Dunn, Rita. (1996). How to Implement and Supervise a Learning Style Program. USA: S.A.C.D. Association for Supervision and Curriculum Development United State.

Gratch, Luis Oscar. (2000). El Trastorno por déficit de atención (ADD-ADHD). Clínica, diagnóstico y tratamiento en la infancia, adolescencia y adultez. Argentina: Editorial Médica Panamericana.

Grigg, Shirley y Rita, Dunn. (1996). Hispannic American Student and Learning Styles USA: ERIC.

Ministerio de Educación Pública. (1997). Políticas, Normativa y Procedimiento para el acceso a la Educación de los Estudiantes con necesidades educativas especiales. Costa Rica: Editorial INA

Revilla, Diana. (1998). Los Estilos de Aprendizaje. Perú: Pontifica Universidad de Perú. Departamento de Educación.

Rojas, Marta, García, Nidia y Brenes, Margarita. (1993). Conocimiento, Participación y Cambio: Tiempo en el aula. San José: Editorial de la Universidad de Costa Rica.

Salas, Raúl. (1995). Aprendiendo y enseñando con estilo. Chile: Ediciones Universidad Austral de Chile. Dirección de Investigación y Desarrollo.

Tannock, Rosemary y Martinussen, Rhonda. (2001). Reconceptualizando el Déficit Atencional. Educational Leadership (pp. 20-25). Traducción libre de M.Ed. Marisol Nieto para uso en la presente investigación.

Wilson, Cynthia. (2002). Learning Styles. Nurturing the genius in each child. Recuperado de http://www.geocities.com/ educationplace/ls.html. 